\title{
Problematischer Umgang mit den Daten der Versicherten
}

\author{
Michel Romanens ${ }^{a}$, Flavian Kurth ${ }^{b}$
}

${ }^{a}$ Dr. med., Präsident Verein Ethik und Medizin Schweiz (VEMS), Mitglied FMH; ${ }^{\text {b }}$ Sekretär Verein Ethik und Medizin Schweiz (VEMS)

Versorgungsforschung ist zentral für Versorgungssicherheit und Versorgungsqualität. Häufig haben Versorgungsstudien aus Kostengründen qualitative Mängel, Zusammenhänge werden überbewertet, Schlussfolgerungen zeigen mehr politisches Wunschdenken als saubere Herleitung. Die Autoren fordern Qualitätslabels in der Versorgungsforschung.

\section{Die Zukunft der Versorgungsforschung?}

Im Artikel «Beratungsresistente Ärzte» in der NZZ vom 11. Januar 2017 zitiert Alain Niederer Prof. Thomas Rosemann, der postuliert, Studien mit Krankenkassendaten seien die Zukunft der Versorgungsforschung. Rosemann fordert: «Die Ärzte sollten sich dieser Möglichkeit nicht verschliessen». Prof. Rosemann spricht hier allerdings von derselben Datenbasis, welche die santésuisse auch zur Beurteilung der Wirtschaftlichkeit der Ärztinnen und Ärzte verwendet und die einen Abdeckungsgrad von 60\% und eine zweifelhafte Qualität hat. So hat der VEMS im Sommer 2016 aufgedeckt, dass darin eine Praxis mit rund 50000 Patienten und rund 30000 Behandlungen erfasst war. Dieser Ausreisser hat dazu geführt, dass irrtümlich zusätzliche $14 \%$ der Ärzte der betreffenden Vergleichsgruppe als auffällig erschienen. Einer davon hat sich an den VEMS

\section{Résumé}

La recherche sur les services de santé est essentielle pour la sécurité et la qualité des soins. Or, les études sur les services de santé sont souvent entachées de défauts qualitatifs; les liens de causalité sont surévalués et les conclusions traduisent davantage des desiderata politiques qu'un souci de rigueur dans les conclusions. Notre revue nous conduit à exiger l'instauration de labels de qualité dans ce type de recherche, ainsi que la publication des données pseudonymes brutes de toutes les banques de données disponibles de I'AOS dans le but de démocratiser la recherche sur les services de santé. gewandt, und wir konnten die statistischen Grundlagen liefern, die santésuisse zur Aufgabe des Verfahrens zwangen. Wären die Kosten des betreffenden Arztes indes mit klinischen Patientendaten bereinigt worden, so hätte sich gezeigt, dass sie medizinisch begründet waren. Solche Fehler sind keine Einzelfälle, sondern systemgegeben, weil die Datenbasis von santésuisse klinisch nicht bereinigt ist. Wenn sich Mediziner gegen Fehlbeurteilungen von Versorgungssituationen durch Studien wehren, die sich auf solche Daten stützen, dann weil sie aufgrund ihrer Praxiserfahrung deren Gefahren für die Patienten sehen.

\section{Eine «Forschung» in der Echokammer}

Es ist an den Studienautoren, ihre Daten klinisch zu bereinigen. Zeigt sich, dass Variablen, welche die vermuteten Zusammenhänge begründen könnten, in der Datenbasis nicht erfasst sind, bleiben diese Zusammenhänge Vermutungen. Diese helfen nicht weiter, und sie werden auch nicht zu Tatsachen, wenn man sie als solche postuliert. Zu welch grotesken Behauptungen dies führen kann, zeigt die jüngste Studie der Helsana zur Versorgung von Diabetes-Patienten [1]. Die Studie kommt entgegen allen nationalen und internationalen Arbeiten zum Thema zum Schluss, bei nicht einmal 5\% der Diabetiker in der Schweiz seien alle notwendigen Kontrollen durchgeführt worden. Der VEMS hat die Studie reviewt und im Dialog mit den Autoren festgestellt: Die Bereinigung der Datenbasis war ungenügend, und dort, wo zum Beispiel auch unter Berücksichtigung soziodemographischer Variablen bereinigt wurde, führte dies zu Feststellungen, die ganz andere Schlüsse verlangt hätten. Diese haben die Autoren aber unterschlagen und aus den unbereinigten Daten ihre 


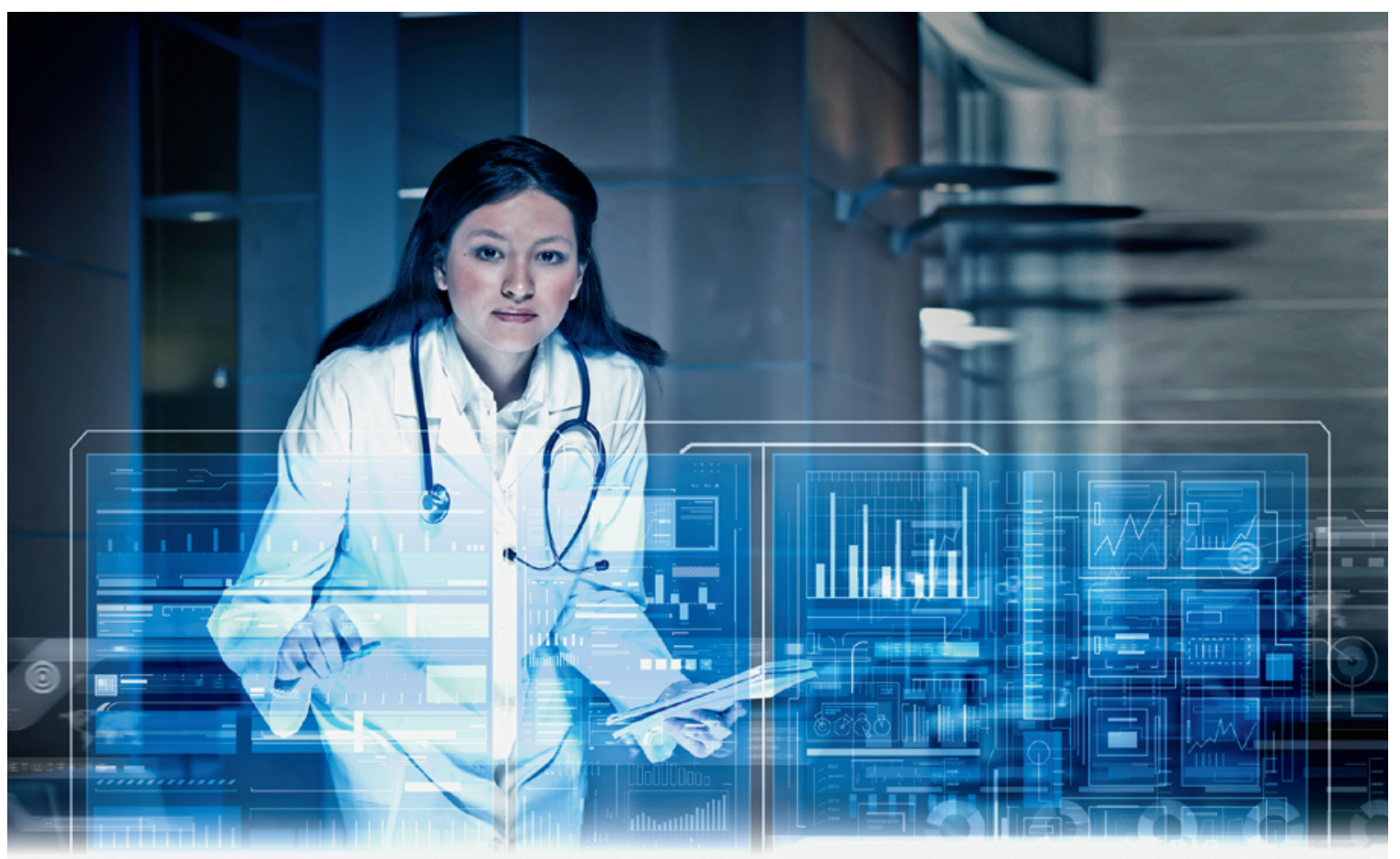

Die Medizin ist gefordert: Im Interesse der Patientinnen und Patienten soll sie auf eine Versorgungsforschung bestehen, die auf medizinischen Fakten basiert.

Aussagen geschlossen. Auf den Einwand hin, die Daten seien nicht bereinigt, können sie nun auf die Tabelle verweisen, wo dies geschehen ist, deren Schlüsse dann aber nicht weiter verfolgt wurden. Eine Salamitaktik. Auch stellt sich die Frage, ob die Daten tatsächlich nicht vorselektiert waren und ausreichend validiert wurden. Wozu also diese Studie? Der CEO der Helsana, Daniel Schmutz, hat sie in einem Gastbeitrag im Tagesanzeiger vom 6. August 2016 mit dem Titel «Die Grenzen der Therapiefreiheit» [2] thematisiert und daraus die Forderung zur Aufhebung des Kontrahierungszwangs abgeleitet. Er beklagt: «Die Gesundheit von $\mathrm{Pa}$ tienten darf nicht länger vom Glück oder Pech in der

\section{Es ist an den Studienautoren, ihre Daten klinisch zu bereinigen.}

Arztwahl abhängen", bedauert, der Helsana seien allerdings die Hände gebunden, und leitet daraus schliesslich seine Forderung ab: «Im Sinne der Patienten und Prämienzahler wäre die beste Option, den Kontrahierungszwang aufzuheben». Jürg Schlup hat diese komplette Fehlinformation mit dem Zweck, die politische Agenda der Helsana zu bedienen, inzwischen in einem Gastbeitrag im Tagesanzeiger richtiggestellt: «Auch die Zahlen der OECD, der Organisation für wirtschaftliche Zusammenarbeit, zeigen, dass die Schweiz als 'top per- former' in der Diabetesbehandlung auf dem zweiten von 31 Länder-Rängen liegt» [3].

\section{Was ist gute Versorgungsforschung?}

Es dürfte konsensfähig sein, dass solche Studien nicht die Zukunft der Versorgungsforschung sein können. So lassen sich keine Erkenntnisse gewinnen, denn solche Studien sind reine Beobachtungsstudien, die Probleme zwar erkennen und Vermutungen anstellen, ein Quantifizieren der Probleme und ein Begründen der Vermutungen aber nicht leisten können. Wie aber sieht eine gute Versorgungsforschung aus? Hierzu liefern die Richtlinien der Good Epidemiological Practice (GEP) eine verbindliche Grundlage, analog zu den Richtlinien der Good Clinical Practice (GCP) für klinische Studien. Während an Letzteren heute kein klinischer Forscher mehr vorbeikommt, verstossen die Versicherer und die von ihnen betrauten und mit ihren Datengrundlagen arbeitenden Institute aber regelmässig gleich in mehreren Punkten gegen Erstere. Qualitativ am höchsten stehen Peer Reviews mit random selection oder Sentinels mit random selection. Beim ersten Typ arbeiten wir mit direkten Arzt-Befragungen und Peer Reviews, basierend auf Fakten mit zufälliger Stichprobe (Zensus-Methode). Alsdann werden die zufälligen Stichproben auf die Allgemeinheit hochgerechnet. 
Auf diese Weise können Sicherheitsfragen und Fragen der Überarztung bzw. der Unterversorgung gut beantwortet werden; der Ansatz gestattet lösungsorientierte Erkenntnisse, die diagnostische Genauigkeit ist hoch. Als Beispiele für solche Studien wären IQM- oder QMMessungen zu erwähnen, allerdings ohne Mortalitätsdaten für Spitäler. Die Sentinel-Methode basiert auf selbst deklarierten Fakten mit zufälliger Stichprobe, ist also eine Kombination von Zensus-Methode und Sentinel-Methode. Auch hier werden die zufälligen Stichproben auf die Allgemeinheit hochgerechnet, was hohe diagnostische Genauigkeit erlaubt. Als Beispiel hierfür ist die Sentinel-Überwachung des Bundes bei den Grippefällen zu nennen.

\section{Was ist schlechte Versorgungsforschung?}

Studien mit individualisierten Versichererdaten sind qualitativ problematischer, da diese Daten auf individualisierten Rechnungsdaten basieren, womit alles, was der Patient aus eigener Tasche bezahlt, nicht erfasst ist - einer der Fehler der Diabetes-Studie der Helsana. Lösungsorientierte Erkenntnisse sind kaum möglich, da die Daten extern nicht überprüft werden können, beispielsweise daraufhin, ob eine Vorselektion vorgenommen wurde. Noch problematischer sind Studien mit aggregierten Versicherten-Daten (SASIS), denn hier kommt als zusätzliches Problem das der Verzerrungen hinzu (Korrelations-Bias, Simpsons-Paradoxon). Die Manipulationsanfälligkeit solcher Studien überbieten einzig noch Befragungen, wie sie beispielsweise Comparis durchführt oder auch der Schweizerische Patientenschutz SKS. Comparis fragt Kunden, die im Managed-Care-Modell sind, ob sie zufrieden seien. Das sind sie auch, wenig erstaunlich, die Prämie ist ja tiefer. Ob sie es auch im Krankheitsfall sind und auch dann, wenn sie chronisch und schwer krank sind, könnte eine systematische Befragung dieser Patientengruppen bringen, insbesondere jener Patienten, die das Modell verlassen. Das tut Comparis aber nicht. Die MC-Forschung der CSS schaut genauer hin, und zwar auf die Behandlungskosten. Steigen diese nach Verlassen des Modells markant, so wäre dies ein Hinweis auf Unterversorgung im Netz. Die CSS untersucht in ihrer Studie «Long-term Effects of Managed Care» [4] von Lukas Kauer nun aber eben nicht die Kosten nach, sondern die bei Verlassen des Modells, stellt fest, dass sie tief sind, und spekuliert, diese Patienten hätten dann wohl in ein noch günstigeres Modell gewechselt. Das ist Kaffeesatzlesen, nicht Wissenschaft. Der Konsumentenschutz SKS schliesslich fragt Patienten, ob sie sich in den letzten Jahren überbehandelt gefühlt hätten [5]. Das ist bei über $23 \%$ der Fall, was sich in etwa mit den immer wieder behaupteten, nie belegten unnötigen Leistungen von angeblich rund 20\% deckt. Daraus irgendetwas zu schliessen, wäre aber so unsinnig, als fragte man Schüler, ob sie sich in den letzten zwei Jahren von ihrer Lehrperson ungerecht behandelt gefühlt hätten, um daraus auf ein Unrecht zu schliessen. Die Antworten könnten auch ein Angleichen an gelernte «Wahrheiten» abbilden. Erinnern sich die Befragten überhaupt an ihre Behandlungen der letzten zwei Jahre? Wurden jene ausgeschlossen, bei denen das nicht der Fall ist?

\section{Beispiel Medikamenteninteraktion}

Dass die Krankenkassen kein Interesse daran haben, eine Forschung zu betreiben, die zum Schluss käme, Hausarztmodelle mit Budgetverantwortung seien nicht für alle Patientengruppen zu empfehlen, ist nachvollziehbar. Dann sollten sie aber auch nicht so tun, als würden sie dies erforschen. Fakt ist: Sie können es gar nicht, denn erst eine Offenlegung der Versichererdaten in anonymisierter Form würde ein Zusammenlegen dieser Daten und damit ein Verfolgen der Kosten über einen Versichererwechsel hinweg erlauben. Auch dass die Versicherer in ihren Studien Unterversorgung ausmachen, wenn sie die Aufhebung des Kontrahierungszwangs beliebt machen wollen, und Überversorgung, haben sie Leistungsabbau im Visier, ist nachvollziehbar, wenn auch nicht eben redlich. Wirklich stossend ist aber, dass die Versicherer dort, wo ihre Daten wichtige Dienste leisten könnten, keine zweckdienliche

\section{Erinnern sich die Befragten überhaupt an ihre Behandlungen der letzten zwei Jahre?}

Forschung betreiben: bei der Identifizierung von Medikamenteninteraktion und Übermedikation. Zu Ersterem: Mit ihren Daten wäre es den Versicherern möglich, Medikamenteninteraktionen vorzubeugen, die passieren können, wenn ein Patient Arzt A verschweigt, dass er von Arzt B ein bestimmtes Medikament bekommt, das mit dem von Arzt A nicht kompatibel ist. Die Kasse weiss von beiden, könnte also reagieren, bevor ein unter Umständen tödliches Ereignis stattfindet. Die Studie «Surveillance of Physicians Causing Potential Drug-Drug Interactions in Ambulatory Care: A Pilot Study in Switzerland" [6] des Basel Institute for Clinical Epidemiology and Biostatistics CEB behauptet, dass gefährliche Medikamenten-Kombinationen erfasst würden, untersucht aber nicht, ob der Arzt durch Aussetzen des einen Medikamentes die Kombination vermieden hat. Ferner wird der Arzt über die ZSR-Nummer anstelle der GLN-Nummer identifi- 
ziert. Die häufigste kontraindizierte Interaktion betraf die Kombination Makrolid-Antibiotika (z.B. Erythromycin) in Kombination mit Statinen. Diese absolute Kontraindikation existiert jedoch gemäss Arzneimittel-Compendium nur für Simvastatin. Bei den übrigen Statinen besteht lediglich eine relative Kontraindikation. Der Marktanteil für Simvastatin betrug im Jahr 2013 20\% (Recherche des Swiss Medical Board 2013). Die Angabe einer DDI von 15/10 000 Patientenjahre betrifft somit lediglich 3/10 000 Patientenjahre, also fünfmal weniger. Dies hat wahrscheinlich auch eine erhebliche Implikation auf die Häufigkeit der DDI bei den Kardiologen. Es bleibt hier also viel Spekulation, und Vorschläge für ein System zur Verhinderung solcher Ereignisse durch Versichererdaten fehlen gänzlich.

\section{Beispiel Übermedikation Antibiotika}

Der Artikel «Beratungsresistente Ärzte» bezieht sich auf eine weitere Studie des CEB auf der Basis von Krankenkassendaten: «Personalized Prescription Feedback Using Routinely Collected Data to Reduce Antibiotic Use in Primary Care: A Randomized Clinical Trial» [7]. Die Autoren suchten aus diesen Daten die 2900 Grundversorger heraus, die am meisten Antibiotika pro 100 Patienten verschreiben, und teilten diese dann zufällig in zwei Gruppen auf, eine, die sie mit Informationen versorgten, und eine, bei der sie dies nicht taten. Resultat: Beide Gruppen haben dennoch weiterhin in etwa gleich oft Antibiotika verschrieben. Schlussfolgerung: Ärzte sind beratungsresistent. Wirklich? Die Arbeit erlaubt es nicht, die Frage zu beantworten, ob die Verwendung von Antibiotika auf der individuellen Ebene angegeben wurde oder nicht. Auch erlaubt sie es nicht, einen Arzt korrekt zu identifizieren. Weiterhin ist nicht klar, wie viele Patienten potentiell unnötige Antibiotika erhielten, da die SASIS-Datenbank nur die Rezeptur von Antibiotika pro 100 Konsultationen für potentiell mehrere Patienten berechnen konnte. Hier wäre mehr zu erfahren, würde man so vorgehen wie unsere Behörden bei der Früherkennung von Epidemien: In den Sentinella-Programmen des Bundes überwachen Ärzte wichtige Aspekte der Volksgesundheit. Ist etwa die Grenze von 60 Grippefällen pro 1000 Konsultationen überschritten, so gilt dies als Epidemie, und es werden entsprechende Massnahmen ergriffen. Es wäre ein Leichtes, auch die Gründe für Antibiotika-Verschreibungen auf realitätsbasierten Sentinella-Erhebungen zu erfassen, und Markus Gnädinger et al. schlagen in ihrer Arbeit «Medication incidents in primary care medicine: protocol of a study by the Swiss Federal Sentinel Reporting System» [8] genau dies vor.

\section{Schlussfolgerung}

Es fällt auch mit viel Wohlwollen schwer, in einer Versorgungsforschung, die so systematisch am Ziel vorbei forscht und so verlässlich die untersuchten Sachverhalte nicht erfasst, eine Methodik zu sehen. Was uns die Versicher als die Zukunft der Versorgungsforschung verkaufen, stimmt wenig zuversichtlich, dass die Hoheit über die Daten der Versicherten bei ihnen in guten Händen ist. Der VEMS fordert, sie anonymisiert öffentlich über die gesamte obligatorische Krankenpflege (OKP) zugänglich zu machen. Ferner sollte ein unabhängiges Gremium die Validität der Aussagen und Schlussfolgerungen gemäss den STROBE-Kriterien [9] überprüfen. Ein Sentinel-System zur Erforschung klinisch relevanter Probleme sollte im Sinne eines Gold-Standards als Grundlage für die Versorgungssicherheit diskutiert werden. Weitere Forschung müsste zunächst klären, ob die Daten der Versicherer überhaupt in der Lage sind, die Signale aus der SentinelGrundlagenforschung korrekt zu erfassen. Solange dies nicht bewiesen ist, sind sie und ihre Institute nicht die geeigneten Organe für die Erfassung, die Erklä-

\section{Schlussfolgerung: Ärzte sind beratungs- resistent. Wirklich?}

rung, die Quantifizierung und die Revalidierung problematischer medizinischer Aktivitäten, zumal sie einseitig wirtschaftliche Eigeninteressen haben. Dass ihre kaum je fruchtbaren, tendenziell aber die Arzt-Patienten-Beziehung verstörenden Studien mit OKP-Geldern finanziert werden, ist ein weiterer problematischer Punkt. Die Medizin ist gefordert, im Interesse der Patientinnen und Patienten auf einer Versorgungsforschung zu bestehen, die auf medizinischen Fakten basiert, nicht auf Vorurteilen, Wunschvorstellungen und wirtschaftlichen Präferenzen der Versicherer.

\section{Bildnachweis}

(c) Sergey Khakimullin

\section{Referenzen}

1 https://www.dovepress.com/a-set-of-four-simple-performancemeasures-reflecting-adherence-to-guid-peer-reviewed-article-PPA

2 http://www.docfind.ch/TADie\%20Grenzender\%20Therapiefrei heit06.08.2016.pdf

3 http://www.docfind.ch/GastbeitragSchlupTAZ092016.pdf.

4 https://www.ncbi.nlm.nih.gov/pubmed/27510575

5 https://www.konsumentenschutz.ch/themen/gesundheitsvor sorge/umfrage-ueberbehandlung-kommunikation-zwischen-arztund-patient-ist-entscheidend/

6 http://journals.plos.org/plosone/article?id=10.1371/journal. pone.0147606

7 www.ncbi.nlm.nih.gov/pmc/articles/PMC4988000/

8 http://bmjopen.bmj.com/content/5/4/e007773.full

9 http://www.strobe-statement.org/fileadmin/Strobe/uploads/ checklists/STROBE_checklist_v4_combined.pdf 